\section{The SPAN III computer program for synthesizing group decisions: Weighting participants' judgments in proportion to confidence}

\author{
WILLIAM J. MACKINNON \\ and \\ LINDA M. ANDERSON \\ University of Arizona \\ Tucson, Arizona 85721
}

A program has been written to extend the capabilities of the Social Participatory Allocative Network (SPAN) method of collecting participants' judgments and synthesizing group decisions. In particular, the SPAN III program can process numbers expressing participants' judgments either with or without processing secondary numbers indicating participants' confidence in their judgments. Thus, it adds confidence weighting to functions performed by two previous versions of the SPAN program.

The initial SPAN program (MacKinnon \& MacKinnon, 1969) processed data obtained by permitting participants to vote both directly for options (alternative solutions to problems, for example) and indirectly by using other members as representatives. The program cumulated the numerical voting power apportioned directly and also iteratively redistributed the indirectly apportioned power according to the receivers' voting pattern, until virtually all power had cumulated for options. The SPAN II program introduced the processing of numerical voting power cast by the method of magnitude estimation (MacKinnon \& Cockrum, 1973). The SPAN III program incorporates these functions of its forerunners.

The weighting extension of the group procedure and computer program for the SPAN III method was designed to improve the quality of group judgments, by utilizing more of the participants' subjective information, and to stimulate members' willingness to participate in complex decision making, by permitting them to specify the weight each of their judgments would carry. To these ends, as SPAN II could be combined with Delphi methods (MacKinnon, 1973), the extended system might also serve in combination.

To specify power, judgment, and confidence, the new system involves for each participant an original quota, two subquotas, four multiple assignments of numbers, and three dual assignments. Allotments of quotas and subquotas and assignments of dual numbers could be divided in various ways between a director and participants, although the program was devised to increase participants' choices. The magnitude estimation method is suitable for the multiple assignments, and the split-100 method for the dual assignments. The following ex- amples of the computation and summary of the program stem, respectively, from unpublished documents by MacKinnon (Note 1) and Anderson (Note 2).

The confidence proportions relating each of the $n$ participants to each of the $\mathrm{m}$ options are given by

$$
c_{i h}=\frac{b_{i h}}{\sum_{h=1}^{m} b_{i h}} \cdot \frac{s_{i}}{g_{i}+s_{i}} \quad i=1,2, \ldots, n ; h=1,2, \ldots, m,
$$

where $b_{i h}$ is the confidence indicator (number) that participant $i$ attaches to option $h, s_{i}$ is the number that member $i$ assigned for confidence allocation to options as a set, and $\mathrm{g}_{\mathrm{i}}$ is the number he assigned for confidence allocation to the members as a group. Likewise, the confidence proportions relating each of the $n$ participants to each of the $n$ participants are given by

$$
c_{i j}=\frac{b_{i j}}{\sum_{j=1}^{n} b_{i j}} \cdot \frac{g_{i}}{g_{i}+s_{i}} \quad i=1,2, \ldots, n ; j=1,2, \ldots, n \text {, }
$$

where $b_{i j}$ is the confidence indicator that participant $i$ attaches to participant $j$. (Nonzero assignments by a participant to himself are ordinarily precluded by instructions.) In Equation 1 the options $h_{1}, h_{2}, \ldots, h_{m}$ correspond to junctions, i.e., options or participants

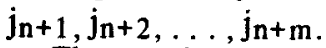

The weighted average number of direct points from all members to each of the $m$ options is found from

$$
\frac{\sum_{i=1}^{n}\left(c_{i h} v_{i}\right)\left(p_{i h} q_{i}\right)}{\sum_{i=1}^{n} c_{i h} v_{i}} \quad h=1,2, \ldots, m,
$$

where $c_{i n}$ is the confidence proportion defined by Equation 1, $v_{i}$ is the confidence quota of participant $i$ and is used for weighting, $p_{i h}$ is the projection proportion relating participant $i$ to option $h$ and is computed as in the SPAN II program, and $q_{i}$ is the magnitude subquota of individual $i$ and is used for judgments. The first pair of parentheses encloses the expression for the weight $\mathrm{w}_{\mathrm{ih}}$, and the second pair contains the expression for the amount of direct power of participant $i$ that was allocated to option $h$ on the first cycle. Correspondingly, the weighted average number of indirect points from all members to each of the $m$ options is found from

$$
\frac{\sum_{i=1}^{n} \sum_{j=1}^{n}\left(c_{i j} v_{i}\right)\left(p_{i j} q_{i} u_{j h}\right)}{\sum_{i=1}^{n} \sum_{j=1}^{n} c_{i j} v_{i}} h=1,2, \ldots, m,
$$


where $c_{i j}$ is the confidence quota defined by Equation 2, $v_{i}$ is the confidence quota of individual $i, p_{i j}$ is the projection proportion relating participant $i$ to participant $\mathrm{j}$ and is computed as in the SPAN II program, $q_{i}$ is the magnitude quota of individual $i, u_{j h}$ is the proportion of member $j$ 's original parcel that accrued for option $h$ directly or indirectly over all cycles and derives from the final cycle of computations of the SPAN II component of the new system, specifically, from the percentage of the cumulative final parcels of member $j$ that is located at junction $h$. The expression within the first pair of parentheses presents the weight $\mathrm{w}_{\mathrm{ij}}$; within the second pair, $p_{i j} q_{i}$ sets forth the amount of indirect power of participant $i$ that was allocated to participant $j$ on the first cycle.

The options for which quantities accrue in accordance with Equations 3 and 4 may be qualitative categories such as applicants for a job, or absolute numbers, e.g., values on a magnitude estimation scale for rating a personality trait. In the absolute case, a different data deck might correspond to each estimated object, and the fractional group quota accruing for each quantitative value produced from a given deck would be used to calculate a weighted average value for the corresponding object.

Limitations. The total number of junctions (members and options combined) must be less than 53 if there are no confidence data, and less than 52 if there are confidence data. Each participant's original quota should not exceed 100 if there are more than 9 group members and should not exceed 1,000 if there are 9 or fewer group members. Each assignment to members and options should be 0 or greater but less than 1,000 .

Computer and Language. SPAN III is written in FORTRAN IV for the Control Data Corporation CDC-6400 computer. The program has a field length requirement of 110,000 (octal) 60-bit words, although this requirement can be reduced by decreasing the number of member and option assignments the program analyzes. The program has been divided into subroutines and extensively commented for easy adaptation to other machines.

Input. The user must prepare a card deck specifying the number of members and number of junctions, the maximum number of iterations requested, the minimum amount of original quotas unallocated to options at the end of the iteration procedure, the number of cards of text he has included in the data, the output options he has selected, and for each participant, his original quota, dual assignment(s), and multiple assignments. Fifty or fewer data decks can be prepared and run in a single execution of the program.

Output. For each data deck, the program prints a title page indicating the size of the group, the number of options, the program options selected by the user, and any text he has included in the data deck. This page is followed by tables repeating the input data, the proportioned data, and the results of the iterations. If confidence data are included, additional tables are produced which repeat the input and proportioned confidence data, and display the weighted direct and indirect points allocated to options. Much of the output is under the control of the user; e.g., he can request the results of iterations for each member individually as well as for the group as a whole.

Avaliability. Copies of the FORTRAN program deck and the SPAN User's Guide are avaliable from William J. MacKinnon, Department of Psychology, University of Arizona, Tucson, Arizona 85721. The charge for mailing both (the program by surface parcel post, the Guide by first-class mail) to addresses within the United States varies between $\$ 2$ and $\$ 8$, depending on location; to addresses outside the United States, the corresponding mailing charge varies between $\$ 11$ and $\$ 14$.

\section{REFERENCE NOTES}

1. MacKinnon, W. J. Prospectus for a weighting process in the SPAN III system. Unpublished manuscript. University of Arizona, July 1974.

2. Anderson, L. M. SPAN user's guide. Unpublished manuscript, University of Arizona, July 1975.

\section{REFERENCES}

MacKinnon, W. J. Weighting for the future: Prospecting the possibilities of weighting Delphi responses with aggregates obtained by the SPAN method. Futures, 1973, 5, 303-308.

MacKinnon, W. J., \& Cockrum, D. L. SPAN II: A modification of the SPAN program for synthesizing group decisions. Behavioral Science, 1973, 18, 78-79.

Mackinnon, W. J., \& MacKinnon, M. M. The decisional design and cyclic computation of SPAN. Behavioral Science, 1969, 14, 244-247. 